\title{
Analysis and Properties of Time-Varying Modified DFT Filter Banks
}

\author{
Guangyu Wang, Zufan Zhang, and Qianbin Chen \\ Chongqing Key Lab of Mobile Communications, Chongqing University of Posts and Telecommunications (CQUPT), \\ Chongqing 400065, China
}

Correspondence should be addressed to Guangyu Wang, wangguangy@cqupt.edu.cn

Received 24 September 2010; Revised 29 November 2010; Accepted 7 December 2010

Academic Editor: Magnus Jansson

Copyright ( 2010 Guangyu Wang et al. This is an open access article distributed under the Creative Commons Attribution License, which permits unrestricted use, distribution, and reproduction in any medium, provided the original work is properly cited.

\begin{abstract}
We provide a comprehensive analysis of the time-varying modified DFT (MDFT) filter bank based on the general time-varying filter bank theories (Wang, 2005, 2006, 2008, 2009) in both the time domain and frequency domain. We give firstly the description of the time-invariant MDFT filter bank including its perfect reconstruction (PR) condition, its mechanism of aliasing error cancellation and the relationship with the cosine-modulated filter bank in detail. Then, the time-varying MDFT filter bank is analyzed according to the time-domain description. Finally, the window switching method is introduced to design the prototype filter in the time-varying MDFT filter bank with examples. The error analysis shows that the introduced design approach is useful in practice.
\end{abstract}

\section{Introduction}

Modified DFT filter banks (MDFT) and their applications are intensively studied in [1-5]. But all analyses are done in the frequency domain for the time-invariant modified DFT filter banks. In the literature, we do not find any works for the time-varying MDFT filter bank. Subband coding is a widely used method in the digital signal processing and the telecommunication areas, such as subband speech and audio coding [5-7], TDM-FDM multiplex [8], DSL communication [9], and subband adaptive filtering [10]. One of the important parts in a subband system is the analysis synthesis filter bank. The analysis filter bank is used to split the input frequency into the different subbands while the synthesis filter bank combines all subbands signals to generate a full band signal.

Among the different subband filter bank structures the modulated filter banks are most popular because of their easy design and efficient implementation. Historically, there are two classes of modulated filter banks, namely, DFT polyphase filter bank and cosine-modulated filter bank. DFT polyphase filter bank was first proposed by Bellangerr and Daguet [8]. DFT polyphase filter bank uses complex modulation. But, the original DFT polyphase filter bank does not provide the mechanism to cancel the adjacent spectrum aliasing. So, in some literatures, the DFT polyphase filter bank was considered as unsuitable for subband applications [11, 12], which also has negative effects on the further research of the DFT polyphase filter bank. To find a method to design the filter bank efficiently, in 1980s the QMF filter bank consisting of two channels and providing the almost perfect reconstruction was introduced. Later, the cosine-modulated filter banks with perfect reconstruction were introduced. Since the introduction, the cosine-modulated filter bank was widely studied and applied due to its perfect reconstruction property and the easy prototype window design with the closed formulation [12]. But, the closed prototype window formulation exists just in the case that the filter length is two times or four times of the channel length, which limits the application of the cosine-modulated filter bank. Without the closed formulation of prototype windows, it is difficult to design an applicable prototype window for a cosinemodulated filter bank to keep the perfect reconstruction property. In this case the cosine-modulated filter bank can only provide an almost perfect reconstruction similar with DFT polyphase filter bank. 
In comparison with cosine-modulated filter banks, the DFT polyphase filter bank has advantages such as linear phase, complex-valued signal processing, and better frequency characteristics in each subband. Furthermore, its computational efficiency is comparable with cosinemodulated filter bank. So, if we can find an approach to efficiently cancel the aliasing error in the DFT polyphase filter bank, the DFT polyphase filter bank has good application possibilities in digital signal processing like speech and image processing. In 1990s, the good application prospects of the DFT polyphase filter bank motivate some researchers to find a mechanism to cancel the aliasing error in the DFT polyphase filter bank. In 1993, Fliege proposed an approach to cancel the aliasing error [1-3]. He called the modified filter bank as modified DFT filter bank (MDFT). The first application of the MDFT filter bank in low-rate speech coding is found in [5], where the MDFT filter bank is used in a $16 \mathrm{kbit} / \mathrm{s}$ speech coder and the simulation showed a good code performance.

The MDFT filter bank proposed by Fliege belongs to time-invariant QMF filter banks. The channel number and subband filters do not change with time. In speech, audio and image processing, the time-varying filter bank can be used to adjust the input signal properties in order to get better processing performance $[6,7,9,10]$. In the past, we have done a systematic research for the general time-varying filter bank including time-varying cosine-modulated filter banks [13-15]. In this paper, we want to use the established timevarying filter bank theory to analyze the time-varying MDFT filter bank (TV-MDFT), and investigate the properties of the MDFT filter bank in the case that its filter coefficients change with time. We want to also study the prototype filter design for a linear time-varying MDFT filter bank. After instruction Section 2 provides the description of the MDFT filter bank and its mechanism of aliasing error cancellation in both the time and frequency domain. Section 3 analyzes the timevarying MDFT filter bank including its PR condition and the relationship with time-varying cosine-modulated filter bank. In Section 4, the design of the prototype window filter for TV-MDFT is discussed. The well-know window switching method used in time-varying cosine-modulated filter bank [13] will be used in the prototype window design for TVMDFT. Section 5 summarizes the main results of the paper.

\section{MDFT Filter Bank}

In [1], the mechanism of the alias cancellation in MDFT filter banks is just described in the frequency domain. Furthermore, the description in [1] seems not to be easy to understand. Here, we try to provide a clear mathematical description of the principle for an MDFT filter bank in both frequency and time domain.

2.1. In the Frequency Domain. Figure 1 shows an $M$-channel MDFT filter bank, where $M$ is even. In the MDFT filter bank, the subband filters in both analysis and synthesis sides are derived from a linear phase FIR prototype filter $H(z)$ by complex modulation. In the analysis side,

$$
H_{k}(z)=H\left(z W_{M}^{k}\right), \quad k=0,1,2, \ldots, M-1 .
$$

The corresponding impulse response reads as

$$
h_{k}(n)=h(n) W_{M}^{-k n}, \quad k=0,1,2, \ldots, M-1,
$$

where $W_{M}^{k}=e^{-j(2 \pi k / M)}$ and $h(n)$ is called prototype window function or prototype filter.

In the synthesis side, the synthesis subband filters are defined similarly as

$$
G_{k}(z)=M H\left(z W_{M}^{k}\right), \quad k=0,1,2, \ldots, M-1 .
$$

The impulse response of the synthesis filters is given by

$$
g_{k}(n)=M h(n) W_{M}^{-k n}, \quad k=0,1,2, \ldots, M-1 .
$$

In order to cancel the aliasing errors in the filter band system showed in Figure 1 and achieve the perfect reconstruction, three key operations are introduced in the MDF filter banks [1].

Operation 1. Band limitation of the prototype filter $H(z)$ to $2 \pi / M$.

Operation 2. Separate each channel into two subchannels with two-step decimation plus interchanged real and imaginary operation.

Operation 3. Introduction of $M / 2$ sample delays in real or imaginary part in each subchannel interchangeably.

Each of the three operations has its own contribution to alias cancellation in the MDF filter bank. The delay of the first subband will be done in the synthesis side, while the delay of the second subchannel in the analysis side. This is the key operation to cancel the adjacent aliasing spectrum.

The band limitation condition can be expressed as

$$
H\left(e^{j \omega}\right)=0, \quad \frac{2 \pi}{M} \leq \omega \leq \pi
$$

The purpose of this condition is to limit the alias errors in the adjacent bands around the analysis subband. In the following, based on the defined condition and key operations we give the detailed analysis of the output-input relationship of an MDFT filter bank in the frequency domain and show how to use the above operations to cancel the alias components in the MDFT filter bank.

It is well known that the output signal $\hat{X}(z)$ in an $M$-channel maximally decimated time-invariant filter bank system can be formulated as

$$
\hat{X}(z)=\frac{1}{M} \sum_{i=0}^{M-1}\left[\sum_{k=0}^{M-1} G_{k}(z) H_{k}\left(z W_{M}^{i}\right)\right] X\left(z W_{M}^{i}\right)
$$




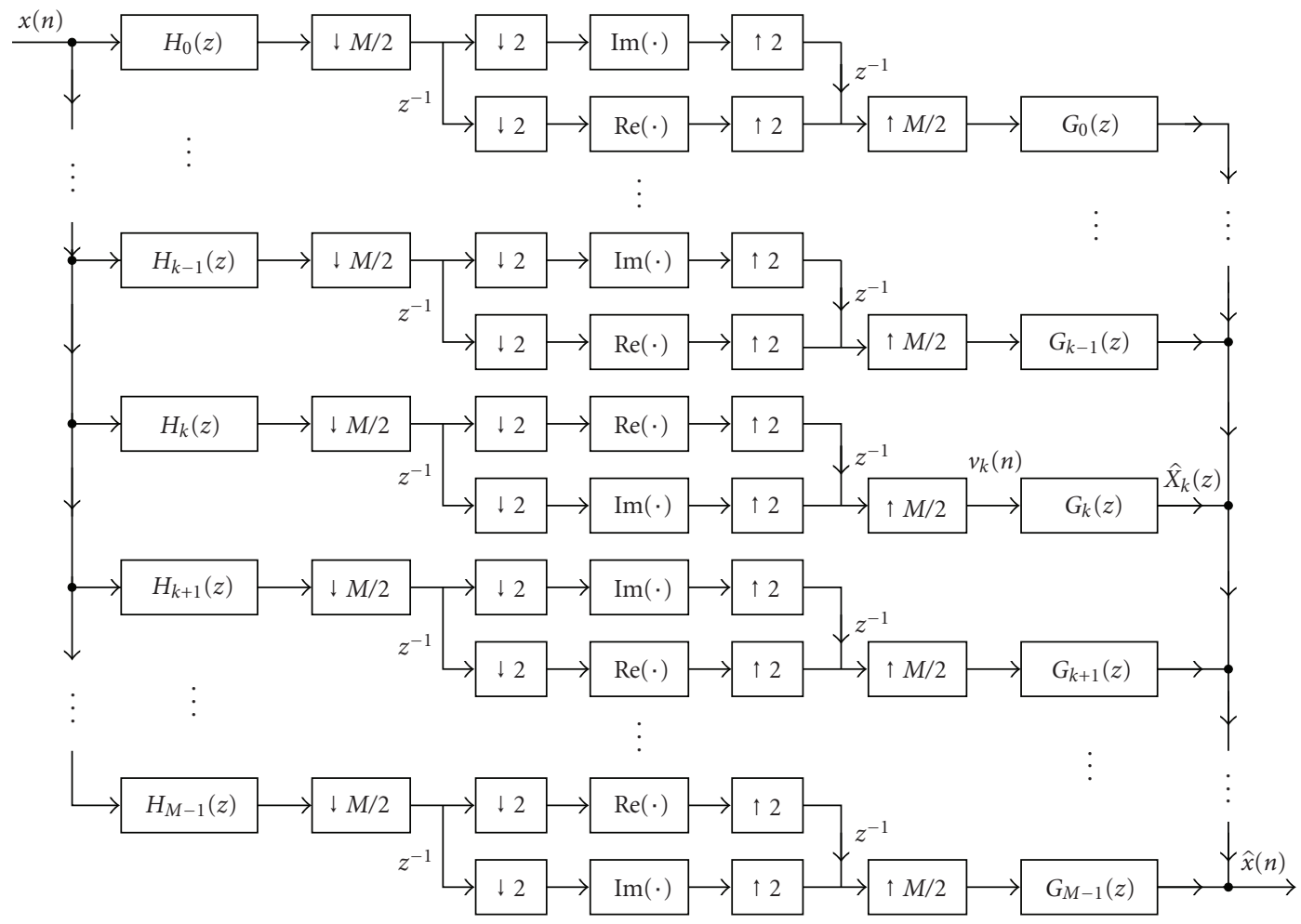

Figure 1: $M$-channel MDFT filter bank, where $M$ is even.

where $k$ indicates the channel number. For an MDFT modulated filter bank, substituting (1) and (3) into (6), we get

$$
\hat{X}(z)=\sum_{i=0}^{M-1}\left[\sum_{k=0}^{M-1} H\left(z W_{M}^{k}\right) H\left(z W_{M}^{k+i}\right)\right] X\left(z W_{M}^{i}\right) .
$$

Using the band limitation condition, (7) can be further simplified as

$$
\begin{gathered}
\hat{X}(z)=\sum_{k=0}^{M-1} H\left(z W_{M}^{k}\right)\left[\sum_{i=-1}^{1} H\left(z W_{M}^{k+i}\right) X\left(z W_{M}^{i}\right)\right] \\
=\frac{1}{M} \sum_{k=0}^{M-1} G_{k}(z)\left[H_{k-1}(z) X\left(z W_{M}^{-1}\right)+H_{k} X(z)\right. \\
\left.+H_{k+1}(z) X\left(z W_{M}^{+1}\right)\right] .
\end{gathered}
$$

From (8), we can see that the contribution of the $k$ th channel to the output signal consists of three parts, the output through $k$ th channel $G_{k}(z) H_{k}(z) X(z)$, the alias component in $(k-1)$ th channel $G_{k}(z) H_{k-1}(z) X\left(z W_{M}^{-1}\right)$, and the alias component in the $(k+1)$ th channel $G_{k}(z) H_{k+1}(z) X\left(z W_{M}^{+1}\right)$.

To cancel the alias components in the adjacent channels, the band limitation is not sufficient. We need still Operations 2 and 3. Operation 2 defines the two-step decimation, real and imaginary operation on the analysis filter output. The goal of the real and imaginary operation is to introduce the mirror spectra in the $k$ th channel. Operation 3 introduces a sign factor $W_{M}^{l M / 2}$ in the first subchannel of each channel in order to move the alias component caused by the adjacent channels. In Figure 1, if we observe the signal $v_{k}(n)$ in the synthesis side and take the $M / 2$ delays in the synthesis side into account, we can write the real part of $v_{k}(n)$ as

$$
\begin{aligned}
v_{k}^{(R)}(n) & =\sum_{i=-\infty}^{\infty} \operatorname{Re}\left\{h_{k}\left(n-\frac{M}{2}-i\right) x(i)\right\} \\
& =\sum_{i=-\infty}^{\infty} \operatorname{Re}\left\{h\left(n-\frac{M}{2}-i\right) W_{M}^{-n k} x(i) W_{M}^{i k}\right\} W_{M}^{k(M / 2)} \\
& =\sum_{i=-\infty}^{\infty} \operatorname{Re}\left\{h\left(n-\frac{M}{2}-i\right) W_{M}^{-n k} x(i) W_{M}^{i k}\right\}(-1)^{k}
\end{aligned}
$$

Considering the fact that the signal $v_{k}(n)$ is got after $M$ downsampling and upsampling, so, just the values of $v_{k}(n)$ at $n=m M$ are not equal to zero and the other values of $v_{k}(n)$ are zero, which means that $W_{M}^{-n k}=1$. Therefore, $v_{k}^{(R)}(n)$ can be expressed as

$$
\begin{aligned}
v_{k}^{(R)}(n) & =\sum_{i=-\infty}^{\infty} h\left(n-\frac{M}{2}-i\right) \operatorname{Re}\left\{x(i) W_{M}^{i k}\right\}(-1)^{k} \\
& =h\left(n-\frac{M}{2}\right) * \operatorname{Re}\left\{x(i) W_{M}^{n k}\right\}(-1)^{k} \\
& =h\left(n-\frac{M}{2}\right) * \frac{1}{2} x(n)\left[W_{M}^{n k}+W_{M}^{-n k}\right](-1)^{k} .
\end{aligned}
$$


In the transform domain, we have

$$
\begin{aligned}
V_{k}^{(R)}(z) & =\frac{1}{2 M} H(z)\left[X\left(z W_{M}^{-k}\right)+X\left(z W_{M}^{k}\right)\right] z^{-M / 2}(-1)^{k} \\
& =\frac{1}{2 M} H_{k}(z)\left[X(z)+X\left(z W_{M}^{2 k}\right)\right] z^{-M / 2}(-1)^{k}
\end{aligned}
$$

Note that the sign factor $(-1)^{k}$ is introduced by $M / 2$ delays in the synthesis side. This sign factor is independent of real and imaginary operation. So, all first subchannels in Figure 1, no matter the real and imaginary parts, have the sign factor $(-1)^{k}$

The imaginary part of $v_{k}(n)$ is coming from the contribution of the second subchannel in the $k$ th channel. From Figure 1, we have the imaginary part of $v_{k}(n)$

$$
\begin{aligned}
v_{k}^{(I)}(n) & =j \sum_{i=-\infty}^{\infty} \operatorname{Im}\left\{h_{k}\left(n-\frac{M}{2}-i\right) x(i)\right\} \\
& =j \sum_{i=-\infty}^{\infty} \operatorname{Im}\left\{h\left(n-\frac{M}{2}-i\right) W_{M}^{-k(n-M / 2)} x(i) W_{M}^{i k}\right\},
\end{aligned}
$$

where $j$ is the imaginary unit. Different from the first subchannel, in the second subchannel of the $k$ th channel, the $M / 2$ delays appear in the output of analysis filters. So, after downsampling and upsampling we get the no-zero values in the second subchannel just at $n-(M / 2)=m M$, which means that $W_{M}^{-k(n-M / 2)}=1$. Considering the $M / 2$ delay effects, we get

$$
\begin{aligned}
v_{k}^{(I)}(n) & =j \sum_{i=-\infty}^{\infty} h\left(n-\frac{M}{2}-i\right) \operatorname{Im}\left\{x(i) W_{M}^{i k}\right\} \\
& =h\left(n-\frac{M}{2}\right) * \frac{1}{2} x(n)\left[W_{M}^{n k}-W_{M}^{-n k}\right] .
\end{aligned}
$$

In the transform domain, we have

$$
V_{k}^{(I)}(z)=\frac{1}{2 M} H_{k}(z)\left[X(z)-X\left(z W_{M}^{2 k}\right)\right] z^{-M / 2},
$$

where there is no sign factor. In general, the second subchannel in Figure 1 has no sign factor.

To declare the mechanism of the adjacent spectrum cancellation in an MDFT filter bank, similar to [1], we suppose that the input spectrum $X\left(e^{j w}\right)$ lies between the center frequency of the $k$ th and $(k+1)$ th channel showed in Figure 2. Note that the position of the input spectrum has no influence on the results.

In Figure 2, the component $X\left(z W_{M}^{2 k}\right)$ describes the mirror spectrum $(\mathbf{M})$ with respect to the original spectrum (O) $X(z)$ about the center frequency of the $k$ th channel because $X\left(e^{j \omega}\right)$ can be written as $X\left(e^{j\left(2 \pi k / M-\omega_{s}\right)}\right)$, while $X\left(e^{j \omega} W_{M}^{2 k}\right)$ is equal to $X\left(e^{-j\left(2 \pi k / M-\omega_{s}\right)}\right)$. AO denotes the alias component due to the shift version of the original spectrum in the $(k-1)$ th channel. AM denotes the alias component caused by the shift version of the mirror spectrum in the $(k+1)$ th channel.

In the following description, we suppose that $k$ is an even number. The result is suitable also for the case of odd $k$. If the $k$ is even, using (11) we get the real part of the $k$ th channel output in Figure 1 as

$$
\begin{aligned}
\hat{X}^{(R)}(z)=\frac{1}{2 M} G_{k}(z)\{ & H_{k}(z) X(z)+H_{k}(z) X\left(z W_{M}^{2 k}\right) \\
& +H_{k}\left(z W_{M}^{-1}\right) X\left(z W_{M}^{-1}\right) \\
& \left.+H_{k}\left(z W_{M}^{+1}\right) X\left(z W_{M}^{2 k+1}\right)\right\} z^{-M / 2}
\end{aligned}
$$

where $H_{k}\left(z W_{M}^{-1}\right) X\left(z W_{M}^{-1}\right)$ is the alias component from the $(k-1)$ th channel $(\mathrm{AO})$, while $H_{k}\left(z W_{M}^{+1}\right) X\left(z W_{M}^{2 k+1}\right)$ is the alias component in the $(k+1)$ th channel (AM). Both parts are coming from the real subchannels in $(k-1)$ th and $(k+1)$ th channels. From Figure 1, we know that the real subchannel in both $(k-1)$ th and $(k+1)$ th channels belongs to second subchannel which has no channel number dependent sign factor because the $M / 2$ delays are done in the analysis filters. So, the sign of the AO and AM components in (15) is positive. Similarly, the imaginary part of $\hat{X}_{k}^{(I)}(z)$ has three contributions from imaginary subchannels in $(k-1)$ th, $k$ th and $(k+1)$ th channels. Based on (14), we have

$$
\begin{aligned}
\hat{X}^{(I)}(z)=\frac{1}{2 M} G_{k}(z)\{ & H_{k}(z) X(z)-H_{k}(z) X\left(z W_{M}^{2 k}\right) \\
& +H_{k}\left(z W_{M}^{-1}\right) X\left(z W_{M}^{-1}\right)(-1)^{k-1} \\
& \left.-H_{k}\left(z W_{M}^{+1}\right) X\left(z W_{M}^{2 k+1}\right)(-1)^{k+1}\right\} z^{-M / 2},
\end{aligned}
$$

where

$$
\begin{aligned}
& \hat{X}_{k, \mathrm{AO}}^{(I)}(z)=H_{k}\left(z W_{M}^{-1}\right) X\left(z W_{M}^{-1}\right)(-1)^{k-1}, \\
& \hat{X}_{k, \mathrm{AM}}^{(I)}(z)=H_{k}\left(z W_{M}^{+1}\right) X\left(z W_{M}^{2 k+1}\right)(-1)^{k+1},
\end{aligned}
$$

are the alias components coming from the imaginary subchannels in $(k-1)$ th and $(k+1)$ th subchannels, respectively. The sign factor is introduced because both imaginary subchannels belong to the second subchannel where the $M / 2$ delays happen in the synthesis side. Up to now, we can better understand why the real and imaginary subchannels must be interchanged. This is exactly the requirement from alias cancellation.

For the even $k$, adding (15) and (16) we get

$$
\begin{aligned}
& \hat{X}_{k}(z) \\
& =\frac{1}{M} G_{k}(z)\left[H_{k}(z) X(z)+H_{k}\left(z W_{k}^{+1}\right) X\left(z W_{M}^{2 k+1}\right)\right] z^{-M / 2} \\
& =\left[H_{k}^{2}(z) X(z)+H_{k}(z) H_{k}\left(z W_{k}^{+1}\right) X\left(z W_{M}^{2 k+1}\right)\right] z^{-M / 2}
\end{aligned}
$$




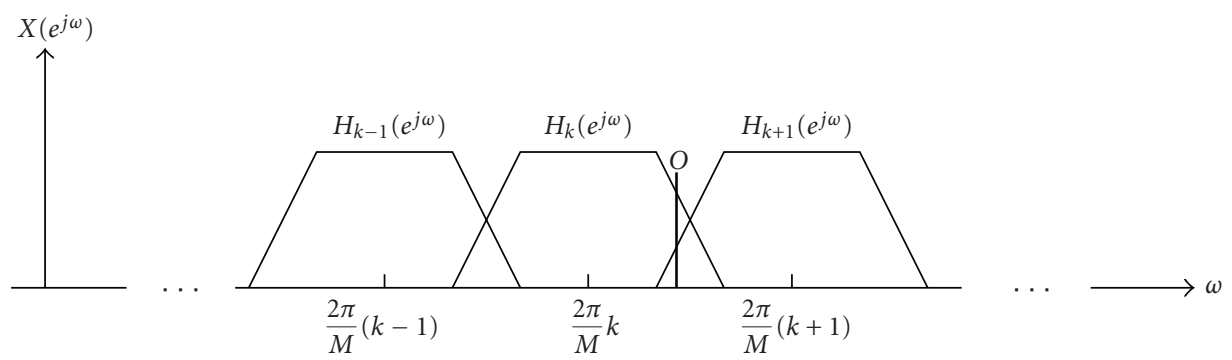

(a) Input spectrum

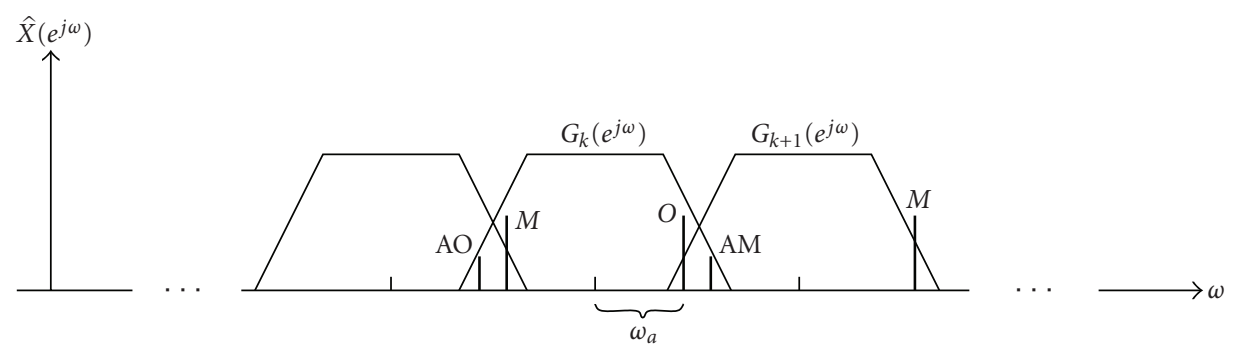

(b) $k$ th channel output spectrum

FIgURE 2: Spectrum change after real and imaginary operation.

In (18), there is still one alias component needed to be canceled, that is, the alias of the mirror spectrum $H_{k}\left(z W_{M}^{+1}\right) X\left(z W_{M}^{2 k+1}\right)$ lying in the $(k+1)$ th channel. Using the similar derivation for $\hat{X}_{k}(z)$, we get the output in the $(k+1)$ th channel for the input $X(z)$ and the mirror spectrum in $(k+1)$ th channel $X\left(z W_{M}^{2 k+2}\right)$ as

$$
\begin{aligned}
\hat{X}_{k+1}(z) \\
=\frac{1}{M} G_{k}(z) \\
\quad \times\left[H_{k+1}(z) X(z)-H_{k+1}\left(z W_{k}^{+1}\right) X\left(z W_{M}^{2(k+1)-1}\right)\right] z^{-M / 2} \\
=\left[H_{k+1}^{2}(z) X(z)-H_{k}(z) H_{k}\left(z W_{k}^{+1}\right) X\left(z W_{M}^{2 k+1}\right)\right] z^{-M / 2},
\end{aligned}
$$

where the mirror alias component has minus sign because the sign factor in(16) is positive after replacing $k$ with $k+1$ in (16), that is, $(-1)^{k}=(-1)^{k+2}=1$. Adding (18), and (19) we get the reconstruction output

$$
\begin{aligned}
\hat{X}(z) & =\hat{X}_{k}(z)+\hat{X}_{k+1}(z) \\
& =X(z)\left[H_{k}^{2}(z)+H_{k+1}^{2}(z)\right] z^{-M / 2} \\
& =X(z)\left[H_{k}^{2}\left(z W_{M}^{k}\right)+H_{k+1}^{2}\left(z W_{M}^{k+1}\right)\right] z^{-M / 2} .
\end{aligned}
$$

Defining

$$
T(z)=H^{2}\left(z W_{M}^{k}\right)+H^{2}\left(z W_{M}^{k+1}\right)
$$

equation (20) can be written as

$$
\hat{X}\left(e^{j \omega}\right)=\left|T\left(e^{j \omega}\right)\right| e^{j(\phi(\omega)+(M / 2) \omega)} X\left(e^{j \omega}\right) .
$$

In the frequency domain, we get

$$
\hat{X}\left(e^{j \omega}\right)=\left|T\left(e^{j \omega}\right)\right| e^{j(\phi(\omega)+(M / 2) \omega)} X\left(e^{j \omega}\right) .
$$

A perfect MDFT filter bank requires that $\left|T\left(e^{j \omega}\right)\right|$ must be constant and $\phi(\omega)$ should be linear function of variable $\omega$. If the prototype filter $H(z)$ has linear phase, we have possibility to satisfy the requirement. Based on [16], a linear phase FIR filter can be described as

$$
H\left(e^{j \omega}\right)=e^{-j \omega(N+1) / 2}\left|H\left(e^{j \omega}\right)\right|,
$$

where $N=r M$ is the filter length, $\left|H\left(e^{j \omega}\right)\right|$ is real function for $\omega$. Using (24), we can write $T\left(e^{j \omega}\right)$ as

$$
\begin{aligned}
T\left(e^{j \omega}\right)=e^{-j(N+1) \omega}[ & \left|H\left(e^{j(\omega-(2 \pi / M) k)}\right)\right|^{2} \\
& \left.+\left|H\left(e^{j(\omega-(2 \pi / M) k-(2 \pi / M)}\right)\right|^{2}\right] .
\end{aligned}
$$

If

$$
\left|H\left(e^{j(\omega)}\right)\right|^{2}+\left|H\left(e^{j(\omega-2 \pi / M)}\right)\right|^{2}=1, \quad 0 \leq \omega \leq \frac{2 \pi}{M},
$$

which means that if the frequency response of the prototype filter is power complementary, we can get the reconstructed signal without distortion. From [16], we know that a linear phase FIR filter can only have two non-zero filter coefficients if the power complementary condition given in (23) must be at the same time satisfied. Such a linear 
phase FIR filter has worse frequency characteristics and is not useful. Another prototype window is introduced in [1], which is derived from an ideal transform function consisting of a square root raised-cosine function and satisfying the power complementary condition (23). But such a prototype window is infinite and noncausal. To make it useful, the truncation and time shift must be done on the prototype window. The finite prototype window is no more power complementary. So, in practice, the condition (23) needs to be given up by designing the prototype filter for an MDFT filter bank. However, it is possible to keep the amplitude distortion arbitrarily small by designing the prototype filter [2].

2.2. In the Time Domain. To make the analysis in the time domain possible, we need to use another structure of the MDFT filter bank, namely, structure with two filter banks without real and imaginary operation shown in Figure 3.

The filter bank in Figure 3 is a $2 M$-channel oversampling filter bank with factor 2 . The analysis filter in $\mathbf{H}^{(1)}(z)$ can be described as

$$
h_{k}^{(1)}(n)=\left\{\begin{aligned}
& j h(n) \operatorname{Im}\left\{W_{M}^{n k}\right\} \\
&= j h(n) \sin \frac{2 \pi}{M} n k, \quad k=0,2, \ldots, \\
& h(n) \operatorname{Re}\left\{W_{M}^{n k}\right\} \\
&=h(n) \cos \frac{2 \pi}{M} n k, \quad k=1,3, \ldots,
\end{aligned}\right.
$$

where $j$ is the imaginary unit. The analysis filters in the
$\mathbf{H}^{(2)}(z)$ have the form

$$
\begin{aligned}
& h_{k}^{(2)}(n) \\
& =\left\{\begin{array}{c}
h\left(n-\frac{M}{2}\right) \operatorname{Re}\left\{W_{M}^{(n-M / 2) k}\right\} \\
=h\left(n-\frac{M}{2}\right) \cos \frac{2 \pi}{M} n k, \quad k=0,2, \ldots, \\
j h\left(n-\frac{M}{2}\right) \operatorname{Im}\left\{W_{M}^{(n-M / 2) k}\right\} \\
=-j h\left(n-\frac{M}{2}\right) \sin \frac{2 \pi}{M} n k, \quad k=1,3, \ldots
\end{array}\right.
\end{aligned}
$$

The synthesis filter is defined by

$$
\begin{aligned}
g_{k}^{(1)}(n) & =M h\left(n-\frac{M}{2}\right) W_{M}^{-k n}(-1)^{k} \\
& =M h\left(n-\frac{M}{2}\right)\left(\cos \frac{2 \pi}{M} k n+j \sin \frac{2 \pi}{M} k n\right)(-1)^{k}, \\
g_{k}^{(2)}(n) & =M h(n) W_{M}^{-k n} \\
& =M h(n)\left(\cos \frac{2 \pi}{M} k n+j \sin \frac{2 \pi}{M} k n\right),
\end{aligned}
$$

where $k=0,1, \ldots, M-1$. Suppose that the analysis and synthesis filters have length $L=N(M / 2)$. In order to describe the MDFT filter bank in the time domain, we need to define the following $M \times(M / 2)$ matrices:

$$
\mathbf{H}^{(j)}(i)=\left[\begin{array}{cccc}
h_{0}^{(j)}\left(L-i \frac{M}{2}+l \frac{M}{2}-1\right) & h_{0}^{(j)}\left(L-i \frac{M}{2}+l \frac{M}{2}-2\right) & \cdots & h_{0}^{(j)}\left(L-i \frac{M}{2}+l \frac{M}{2}-\frac{M}{2}\right) \\
h_{1}^{(j)}\left(L-i \frac{M}{2}+l \frac{M}{2}-1\right) & h_{1}^{(j)}\left(L-i \frac{M}{2}+l \frac{M}{2}-2\right) & \cdots & h_{1}^{(j)}\left(L-i \frac{M}{2}+l \frac{M}{2}-\frac{M}{2}\right) \\
\vdots & \vdots & \vdots \\
h_{M-1}^{(j)}\left(L-i \frac{M}{2}+l \frac{M}{2}-1\right) & h_{M-1}^{(j)}\left(L-i \frac{M}{2}+l \frac{M}{2}-2\right) & \cdots & h_{M-1}^{(j)}\left(L-i \frac{M}{2}+l \frac{M}{2}-\frac{M}{2}\right)
\end{array}\right],
$$

$$
\mathbf{H}^{(1)}(i)=\left[\begin{array}{cccc}
g_{0}^{(1)}\left(i \frac{M}{2}+\frac{M}{2}\right) & g_{1}^{(1)}\left(i \frac{M}{2}+\frac{M}{2}\right) & \cdots & g_{M-1}^{(1)}\left(i \frac{M}{2}+\frac{M}{2}\right) \\
g_{0}^{(1)}\left(i \frac{M}{2}+\frac{M}{2}+1\right) & g_{1}^{(1)}\left(i \frac{M}{2}+\frac{M}{2}+1\right) & \cdots & g_{M-1}^{(1)}\left(i \frac{M}{2}+\frac{M}{2}+1\right) \\
\vdots & \vdots & \vdots \\
g_{0}^{(1)}\left(i \frac{M}{2}+\frac{M}{2}+\frac{M}{2}-1\right) & g_{1}^{(1)}\left(i \frac{M}{2}+\frac{M}{2}+\frac{M}{2}-1\right) & \cdots & g_{M-1}^{(j)}\left(i \frac{M}{2}+\frac{M}{2}+l \frac{M}{2}-1\right)
\end{array}\right],
$$




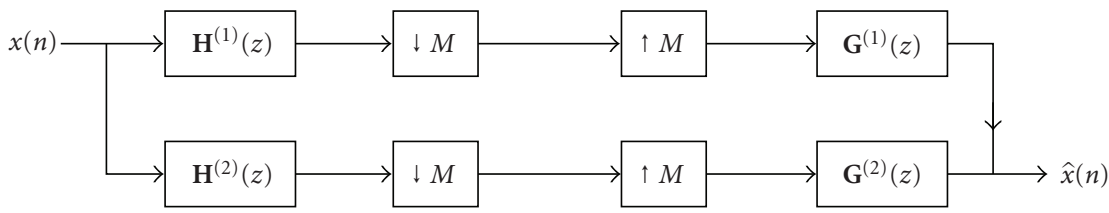

FIGURE 3: Implementation of the MDFT filter bank as two $M$-channel filter banks.

$$
\mathbf{G}^{(2)}(i)=\left[\begin{array}{cccc}
g_{0}^{(2)}\left(i \frac{M}{2}\right) & g_{1}^{(2)}\left(i \frac{M}{2}\right) & \cdots & g_{M-1}^{(2)}\left(i \frac{M}{2}\right) \\
g_{0}^{(2)}\left(i \frac{M}{2}+1\right) & g_{1}^{(2)}\left(i \frac{M}{2}+1\right) & \cdots & g_{M-1}^{(j)}\left(i \frac{M}{2}+l \frac{M}{2}+1\right) \\
\vdots & \vdots & \vdots \\
g_{0}^{(2)}\left(i \frac{M}{2}+\frac{M}{2}-1\right) & g_{1}^{(2)}\left(i \frac{M}{2}+\frac{M}{2}-1\right) & \cdots & g_{M-1}^{(2)}\left(i \frac{M}{2}+\frac{M}{2}-1\right)
\end{array}\right],
$$

where $i=0,1, \ldots, N-1, j=1,2$, and $l=\operatorname{floor}(j / 2)$.

For analysis of the MDFT filter bank in the time domain, we define two infinite matrices $\mathbf{T}_{a}$ and $\mathbf{T}_{s}$ as

$$
\mathbf{T}_{a}=\left[\begin{array}{ccccccccc} 
& \vdots & \vdots & \vdots & \vdots & \vdots & \vdots & \vdots & \\
\cdots & \mathbf{0} & \mathbf{H}^{(1)}(0) & \mathbf{H}^{(1)}(1) & \mathbf{H}^{(1)}(2) & \cdots & \mathbf{H}^{(1)}(N-2) & \mathbf{H}^{(1)}(N-1) & \cdots \\
\cdots & \mathbf{H}^{(2)}(0) & \mathbf{H}^{(2)}(1) & \mathbf{H}^{(2)}(2) & \mathbf{H}^{(2)}(3) & \cdots & \mathbf{H}^{(2)}(N-1) & \mathbf{0} & \cdots \\
\ldots & \cdots & \ldots & \mathbf{0} & \mathbf{H}^{(1)}(0) & \cdots & \mathbf{H}^{(1)}(N-4) & \mathbf{H}^{(1)}(N-3) & \cdots \\
\cdots & \cdots & \ldots & \mathbf{H}^{(2)}(0) & \mathbf{H}^{(2)}(1) & \cdots & \mathbf{H}^{(2)}(N-3) & \mathbf{H}^{(2)}(N-2) & \cdots \\
& \vdots & \vdots & \vdots & \vdots & \vdots & \vdots & \vdots
\end{array}\right],
$$

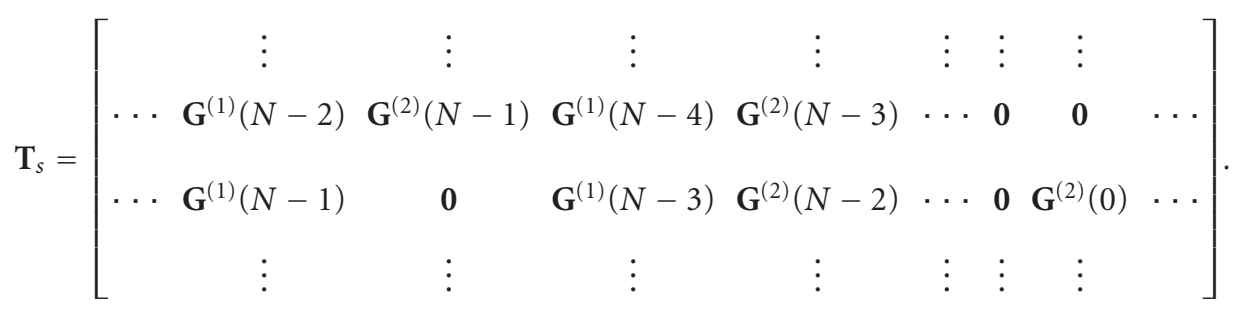

$\mathbf{T}_{a}$ and $\mathbf{T}_{s}$ are called analysis and synthesis matrices and describe the relationship of the MDFT analysis and synthesis filter bank coefficients during the filter bank operation in the time domain. These two infinite matrices are the basis for the analysis of MDFT filter bank in the time domain. Using the matrices (31)-(33) and (34)-(35), we can describe the MDFT filter bank operation in the time domain as

$$
\widehat{\mathbf{x}}=\mathbf{T}_{s} \mathbf{T}_{a} \mathbf{x}
$$


where $\mathbf{x}$ and $\hat{\mathbf{x}}$ are the input and output vectors defined, respectively, by

$$
\begin{aligned}
& \mathbf{x}=\left[\begin{array}{lllll}
\cdots & x(-1) & x(0) & x(1) & \cdots
\end{array}\right]^{T}, \\
& \hat{\mathbf{x}}=\left[\begin{array}{lllll}
\cdots & \hat{x}(-1) & \hat{x}(0) & \hat{x}(1) & \cdots
\end{array}\right]^{T} .
\end{aligned}
$$

It is clear that the perfect reconstruction (PR) condition for the MDFT filter bank is

$$
\mathbf{T}_{s} \mathbf{T}_{a}=\mathbf{I},
$$

where I is an infinite unit matrix. Together with (26), we have the following theorem.

Theorem 1. The following $P R$ conditions are equivalent for an MDFT filter bank

(1) $\left|H\left(e^{j \omega}\right)\right|^{2}+\left|H\left(e^{j(\omega-(2 \pi / M))}\right)\right|^{2}=1,0 \leq \omega \leq 2 \pi / M$,

(2) $\mathbf{T}_{a}^{T} \mathbf{T}_{a}=\mathbf{I}$.

2.3. Relationship with Cosine-Modulated Filter Bank. If we remove the imaginary channels in Figure 1, the channel number in $\mathbf{H}^{1}(z)$ and $\mathbf{H}^{2}(z)$ reduces to $M / 2$. Then, an MDFT filter bank becomes a critically decimated $M$-channel filter bank with real filter coefficients. The filter definitions in (27) and (28) can be simplified as

$$
\begin{array}{ll}
h_{k}^{(1)}(n)=h(n) \cos \phi_{k}(n), & k=1,3, \ldots, \\
h_{k}^{(2)}(n)=h(n) \cos \phi_{k}(n), & k=0,2, \ldots,
\end{array}
$$

where $\phi_{k}(n)=(2 \pi / M) n k$. After removing the imaginary channels in the MDFT filter bank, the mechanism described above for alias cancellation does not work, because the sign factor introduced by $M / 2$ delays has no contribution to alias cancellation. So, the two stages sampling and $M / 2$ delays make no sense. Removing the delay $z^{-M / 2}$ in Figure 3, we get an $M$-channel cosine-modulated filter bank in Figure 4.

The filters in Figure 4 are defined as

$$
\begin{gathered}
h_{k}(n)=h(n) \cos \phi_{k}(n), \quad 0 \leq k \leq M-1, \\
g_{k}(n)=M h(n) \cos \phi_{k}(n), \quad 0 \leq k \leq M-1 .
\end{gathered}
$$

From [11, 13], we know that it is possible to get a perfectly reconstructed cosine-modulated filter bank if the phase $\phi_{k}(n)$ is properly selected. It is clear that the phase $\phi_{k}(n)=(2 \pi / M) n k$ does not get perfect reconstruction. One possible $\phi_{k}(n)$ that leads to a perfectly reconstructed cosinemodulated filter bank is [13]

$$
\phi_{k}(n)=\cos \left[\left(k+\frac{1}{2}\right)\left(n+\frac{M+1}{2}\right) \frac{\pi}{M}\right] .
$$

\section{Design of MDFT Filter Bank}

The design of the MDFT filter bank is based on Theorem 1. Using the PR condition (2) in Theorem 1, the design can be formulated as the following equation

$$
\mathbf{e}=\mathbf{T}_{a}^{T} \mathbf{T}_{a}-\mathbf{I},
$$

where $\mathbf{e}$ is the error vector. The design is an optimization process to find the filter coefficients in $\mathbf{T}_{s}$ at the minimal error point. This optimization needs a large computation efforts. So, it is not the first choice for the MDFT filter bank design.

Another method is to use the PR condition (1) in Theorem 1. Here we can use the design approach of the FIR filter, where the filter coefficients of an ideal low-pass filter are first got through inverse transform. Then, the FIR filter coefficients are attained by windowing the infinite idea low pass filter coefficients. A possible ideal prototype filter satisfying the power complementary is the square root raised-cosine function defined by [2]

$$
\begin{aligned}
& H_{d}(j \omega) \\
& = \begin{cases}1, & |\omega|<(1-r) \omega_{c} \\
\frac{1}{2}+ & \frac{1}{2} \cos \left(\frac{\pi}{2 r}\left(\frac{\omega}{\omega_{c}}+r-1\right)\right), \\
& (1-r) \omega_{c} \leq \omega \leq(1+r) \omega_{c} \\
0, & |\omega|>(1+r) \omega_{c}\end{cases}
\end{aligned}
$$

where $\omega_{c}$ is the cut-off frequency. For an MDFT filter bank, $\omega_{c}$ is equal to $2 \pi / M . r$ is the roll-off factor $(0<r \leq 1)$ and decides the weight of transient band of the filter. $H_{d}(j \omega)$ is ideal for an MDFT filter bank because

$$
\left|H_{d}(j \omega)\right|^{2}+\left|H_{d}\left(j\left(\omega-\frac{2 \pi}{M}\right)\right)\right|^{2}=1, \quad 0 \leq \omega \leq \frac{2 \pi}{M} .
$$

Through inverse transform of $H_{d}(j \omega)$ we have the impulse response function of the prototype filter as [2]

$$
\begin{aligned}
& h_{d}(n) \\
& =\frac{(4 r n / M) \cos (\pi(1+r) n / M)+\sin (\pi(1-r) n / M)}{\left(1-(4 r n / M)^{2}\right)(4 r n / M+(\pi(1-r) n / M))}, \\
& -\infty<n<\infty .
\end{aligned}
$$

An MDFT filter bank with $h_{d}(n)$ has perfect reconstruction. But $h_{d}(n)$ is infinite and noncausal. So, $h_{d}(n)$ is unrealisable. To get the realisable and causal prototype filter, we can simply truncate and shift the function $h_{d}(n)$. After truncating and time shifting, we have

$$
h(n)=h_{d}(n-N M) w(n-N M),
$$

where $N M$ is the prototype filter length. From [2], we know that it is possible to keep the aliasing distortion arbitrarily small by selecting the roll-off factor $r$ and the window function $w(n)$. Figure 5 shows the examples of the transfer function $H_{d}(j \omega)$ and its corresponding truncatingimpulse 


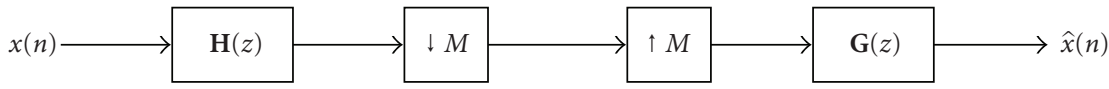

FIGURE 4: An $M$-channel cosine-modulated filter bank.

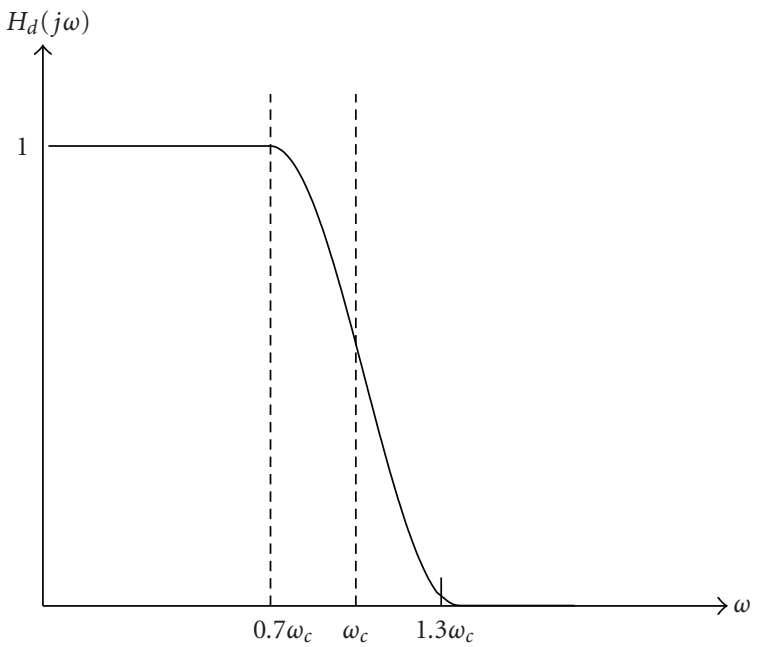

(a) $H_{d}(j \omega)$ with $r=0.3$

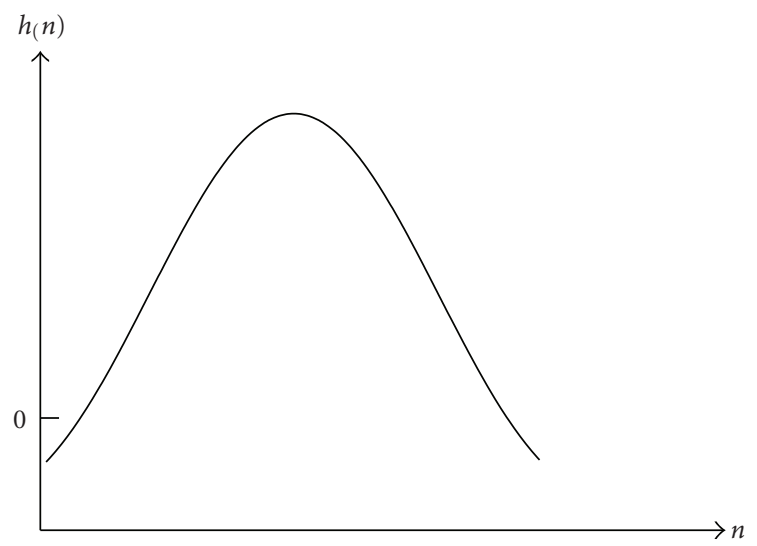

(c) $h(n)$ with $N=2 M, r=0.3, w(n)=1$

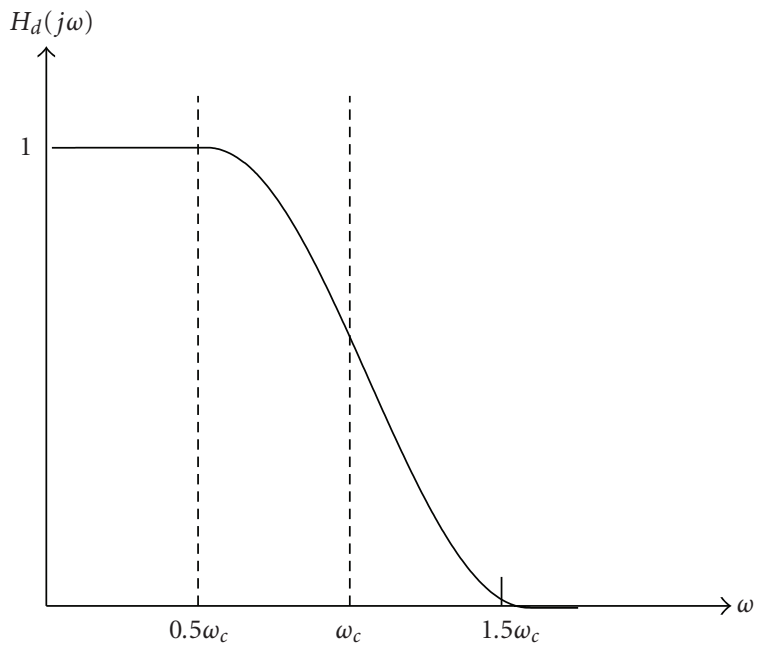

(b) $H_{d}(j \omega)$ with $r=0.5$

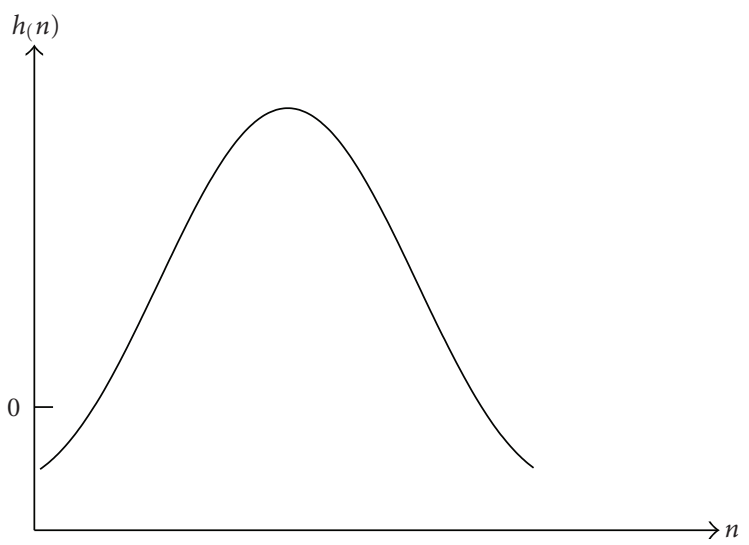

(d) $h(n)$ with $N=2 M, r=0.5, w(n)=1$

FIGURE 5: Examples of ideal prototype filter $H_{d}(j \omega)$ and its deduced prototype filter coefficients $h(n)$ at different roll-off factor $r$.

response function $h(n)$ with roll-off factor $r=0.3$ and $r=0.5$, respectively.

\section{Time-Varying MDFT Filter Bank}

The general time-varying filter bank is studied in $[14,15]$. In [13], the time-varying cosine-modulated filter bank is analyzed. The window switching methods are introduced to design the time-varying cosine-modulated filter bank. The MDFT filter bank is same as the cosine-modulated filter bank in that both filter banks are got through modulation of a prototype filter. The difference is that cosine-modulated filter banks use a cosine function as the modulation function while the modulator in the MDFT filter bank is complex valued. So, the window switching methods can also be used in design of MDFT filter banks. In the following, we give firstly the description of a time-varying MDFT filter bank with focus on an $M$-channel time-varying filter bank. Then, the window switching methods are used to design the $M$-channel timevarying filter bank.

4.1. Description of Time-Varying MDFT Filter Bank. Similar to that in [15], we define an $M$-channel time-varying MDFT filter bank as the MDFT filter bank whose analysis and synthesis filter coefficients are varying with the index $m$, where $m=$ floor $(n / M)$ is the integer part of $n / M$. Figure 6 shows an $M$-channel time-varying MDFT filter bank.

The filter coefficients in (27)-(28) become

$$
\begin{aligned}
& h_{k}^{(1)}(n, m) \\
& = \begin{cases}j h(n, m) \sin \frac{2 \pi}{M} n k, & k=0,2, \ldots, \\
h(n, m) \cos \frac{2 \pi}{M} n k, & k=1,3, \ldots,\end{cases}
\end{aligned}
$$




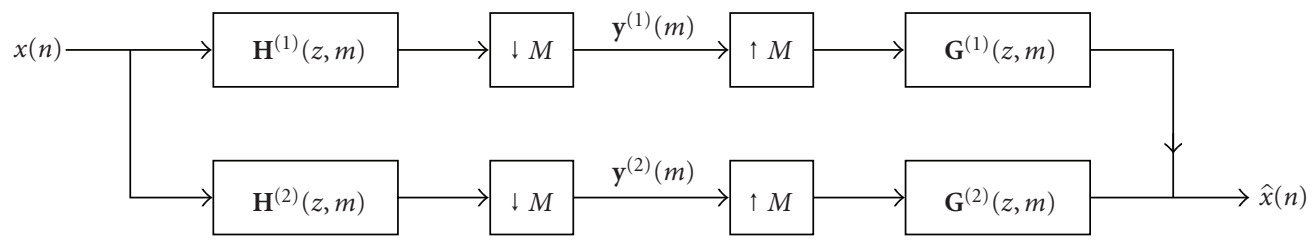

Figure 6: $M$-channel time-varying MDFT filter bank.

$$
\begin{aligned}
& h_{k}^{(2)}(n, m) \\
& = \begin{cases}h\left(n-\frac{M}{2}, m\right) \cos \frac{2 \pi}{M} n k, & k=0,2, \ldots, \\
-j h\left(n-\frac{M}{2}, m\right) \sin \frac{2 \pi}{M} n k, & k=1,3, \ldots,\end{cases}
\end{aligned}
$$

where $h(n, m)$ is the time-varying prototype filter. $\mathbf{H}^{(1)}(i, m)$ is defined as

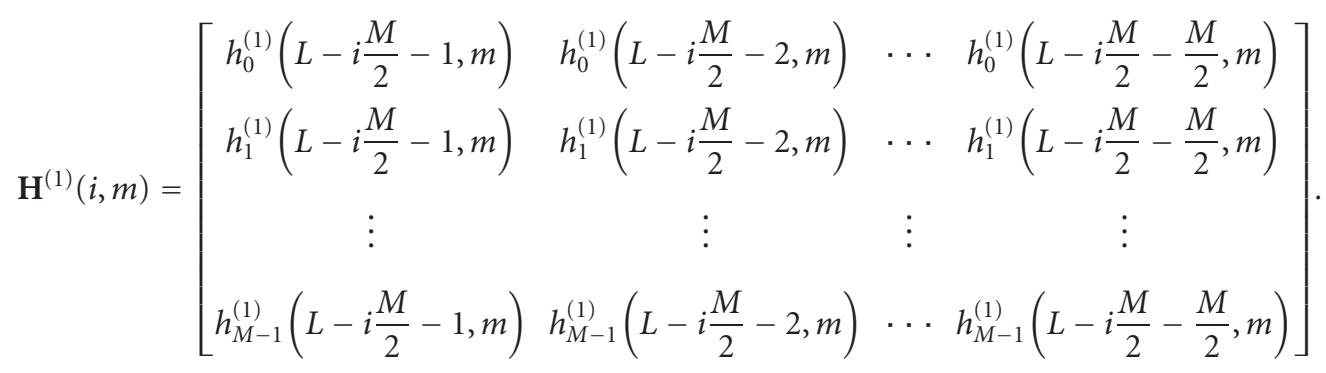

$\mathbf{H}^{(2)}(i, m)$ and $\mathbf{G}(i, m)$ have the similar definitions. The timevarying analysis matrix $\mathbf{T}_{a}(m)$ has the form

$$
\mathbf{T}_{a}(m)=\left[\begin{array}{cccccccc} 
& \vdots & \vdots & \vdots & \vdots & \vdots & \vdots \\
\ldots & \mathbf{0} & \mathbf{H}^{(1)}(0, m-1) & \mathbf{H}^{(1)}(1, m-1) & \mathbf{H}^{(1)}(2, m-1) & \cdots & \mathbf{H}^{(1)}(N-1, m-1) & \cdots \\
\cdots & \mathbf{H}^{(2)}(0, m-1) & \mathbf{H}^{(2)}(1, m-1) & \mathbf{H}^{(2)}(2, m-1) & \mathbf{H}^{(2)}(3, m-1) & \ldots & \mathbf{0} & \cdots \\
\ldots & \ldots & \ldots & \mathbf{0} & \mathbf{H}^{(1)}(0, m) & \ldots & \mathbf{H}^{(1)}(N-3, m) & \cdots \\
\ldots & \ldots & \ldots & \mathbf{H}^{(2)}(0, m) & \mathbf{H}^{(2)}(1, m) & \ldots & \mathbf{H}^{(2)}(N-2, m) & \cdots \\
\vdots & \vdots & \vdots & \vdots & \vdots & \vdots
\end{array}\right] .
$$

Similarly, replacing $\mathbf{G}^{(1)}(i)$ and $\mathbf{G}^{(2)}(i)$ with $\mathbf{G}^{(1)}(i, m)$ and $\mathbf{G}^{(2)}(i, m)$ in (35) we have the time-varying synthesis matrix $\mathbf{T}_{s}(m)$

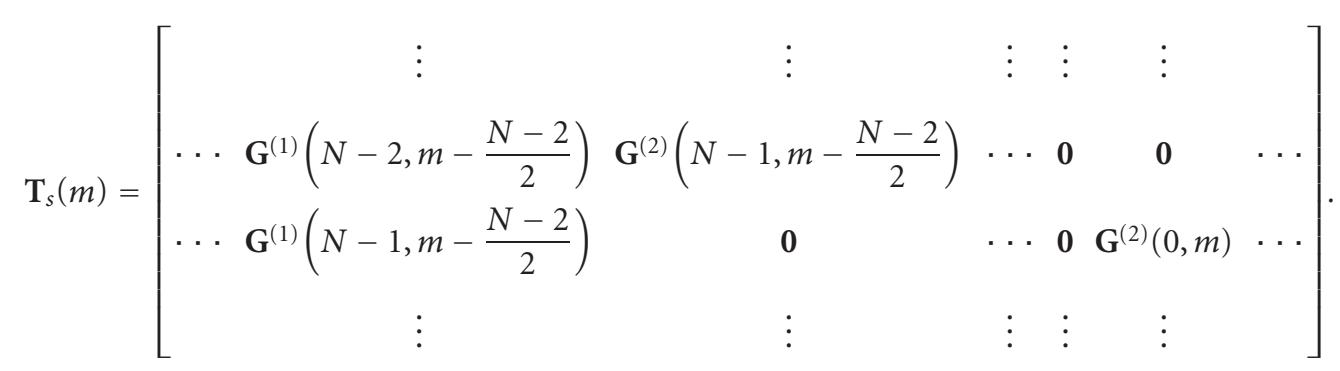


The PR condition of the time-varying MDFT filter bank is

$$
\mathbf{T}_{s}(m) \mathbf{T}_{a}(m)=\mathbf{I} .
$$

The power complementary condition at each time index $m$ does not make sure that the time-varying MDFT filter bank has a PR property. We know that the convolution rule in a time-invariant system does not exist in a time-varying system [14]. So, the frequency-domain analysis method for the aliasing cancellation described in Section 2 cannot be used for analyzing the time-varying MDFT filter banks. The time-domain analysis method is more efficient for the timevarying filter bank analysis and design.

4.2. Design of Time-Varying MDFT Filter Banks. The basis of filter bank design is its PR condition. In the time-invariant case, we can use the power complementary condition (38) to design the time-invariant MDFT filter bank. But, the power complementary condition does not exist for the time-varying MDFT filter bank. So, the only useful design condition is the PR condition (52). The general design approach for a time-varying filter bank is introduced in [17]. In [17], the least squares (LS) solution is provided to calculate the synthesis filter coefficients in the transient period from one MDFT filter bank to another MDFT filter bank. But, the LS solution needs the calculation of the inverse matrix. In [13], a window switching method is introduced to design the timevarying cosine-modulated filter bank, where the prototype filter impulse response function or the prototype window function in the transient period is got through changing the pieces of window based on the prototype windows before and after changing at the time $m$. The window switching method does not need any calculation. It is quite useful for the design of time-varying filter bank with modulation of the prototype filter because in such a filter bank system the filter coefficients are described by prototype filter. We just need to design the prototype window function at each time index $m$. In the following, we give an example to describe how to design the time-varying MDFT filter bank using window switching methods.

Before using window switching methods to design the prototype filter, we need to know the restrictions that the PR condition imposes on the prototype filter $h(n, m)$. We define following matrices:

$$
\begin{gathered}
\boldsymbol{\Phi}^{(1)}=\left[\begin{array}{llll}
\boldsymbol{\Phi}_{0}^{(1)} & \boldsymbol{\Phi}_{1}^{(1)} & \cdots & \boldsymbol{\Phi}_{N-1}^{(1)}
\end{array}\right] \\
\boldsymbol{\Phi}^{(2)}=\left[\begin{array}{llll}
\boldsymbol{\Phi}_{0}^{(2)} & \boldsymbol{\Phi}_{1}^{(2)} & \cdots & \boldsymbol{\Phi}_{N-1}^{(2)}
\end{array}\right] \\
\mathbf{H}(m)=\operatorname{diag}\left[\begin{array}{llll}
\mathbf{H}_{0}(m) & \mathbf{H}_{1}(m) & \cdots & \mathbf{H}_{N-1}(m)
\end{array}\right]
\end{gathered}
$$

where

$$
\begin{gathered}
{\left[\boldsymbol{\Phi}_{i}^{(1)}\right]_{k n}=\sqrt{\frac{2}{M}} \sin \left(\frac{2 \pi}{M} n k\right), \quad k=0,2, \ldots, \frac{M-2}{2},} \\
{\left[\boldsymbol{\Phi}_{i}^{(1)}\right]_{k n}=(-1)^{i} \sqrt{\frac{2}{M}} \cos \left(\frac{2 \pi}{M} n k\right), \quad k=1,3, \ldots, \frac{M-1}{2},}
\end{gathered}
$$

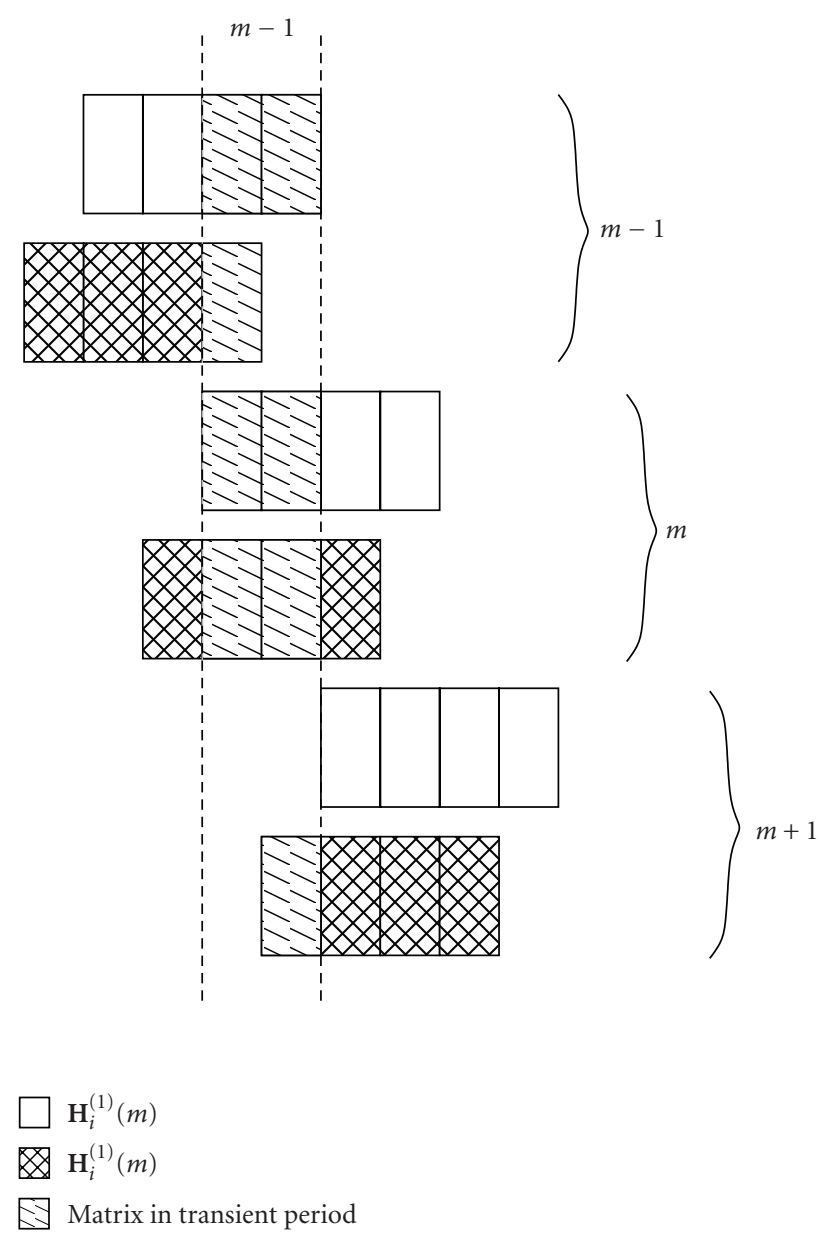

FIgURE 7: Graphical representation of the analysis matrix $\mathbf{T}_{a}(m)$ around time point $m$.

$$
\left[\boldsymbol{\Phi}_{i}^{(2)}\right]_{k n}=\sqrt{\frac{2}{M}} \cos \left(\frac{2 \pi}{M} n k\right), \quad k=0,2, \ldots, \frac{M-2}{2},
$$

$$
\left[\Phi_{i}^{(2)}\right]_{k n}=(-1)^{i+1} \sqrt{\frac{2}{M}} \sin \left(\frac{2 \pi}{M} n k\right), k=1,3, \ldots, \frac{M-1}{2},
$$

$$
\begin{gathered}
\mathbf{H}_{i}(m)=\operatorname{diag}\left[h\left(L-i \frac{M}{2}-1, m\right) h\left(L-i \frac{M}{2}-2, m\right)\right. \\
\left.\cdots h\left(L-i \frac{M}{2}-\frac{M}{2}+1, m\right)\right],
\end{gathered}
$$

where $i=0,1, \ldots, N-1, n=0,1, \ldots, M / 2-1$, and $k=$ $0,1, \ldots, M-1$.

Using matrices (54)-(58), we can write the analysis matrix in (48) as

$$
\mathbf{H}^{(1)}(i, m)=\Phi_{i}^{(1)} \mathbf{H}_{i}(m)
$$




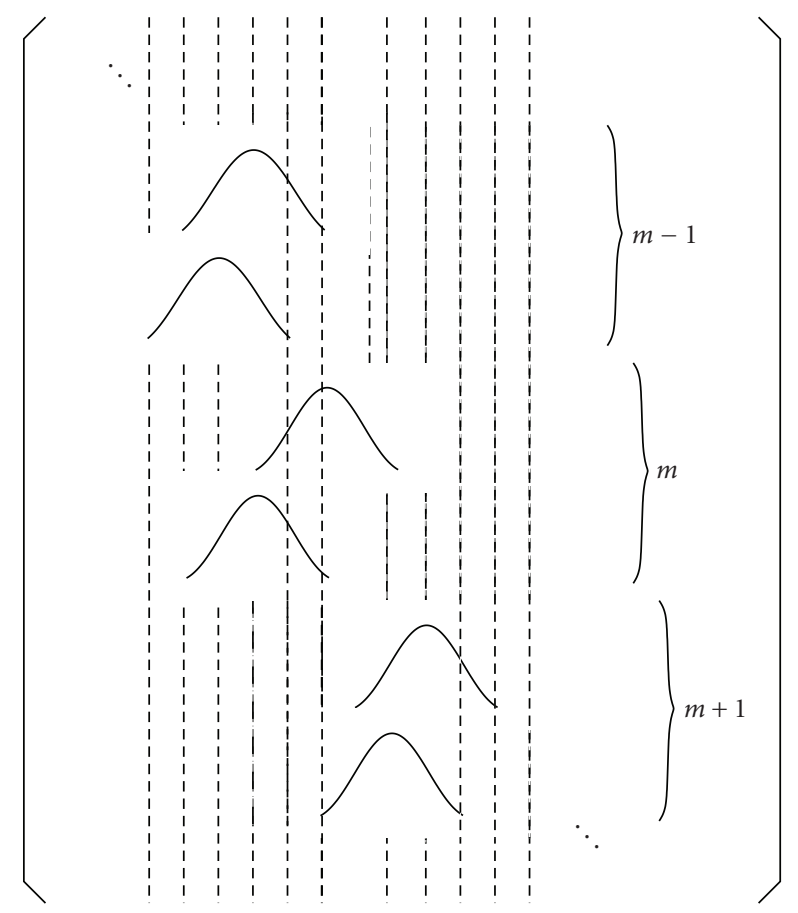

FIgURE 8: Window matrix for Figure 7. where $N=2 K, s=0,1, \ldots, K-1$. I is the $(M / 2) \times(M / 2)$ unit matrix. Substituting (61)-(62) into (63)-(64), we have

$$
\begin{aligned}
& \sum_{i=0}^{K-s-1} \mathbf{H}_{N-1-2 i}(m+i)\left[\boldsymbol{\Phi}_{N-1-2 i}^{(2)}\right]^{T}\left[\boldsymbol{\Phi}_{N-1-2 i-s}^{(2)}\right] \\
& \times \mathbf{H}_{N-1-2 \mathrm{i}-s}(m+i) \\
& +\mathbf{H}_{N-2 i-2}(m+i)\left[\boldsymbol{\Phi}_{N-2 i-2}^{(1)}\right]^{T}\left[\boldsymbol{\Phi}_{N-2 i-2-s}^{(1)}\right] \mathbf{H}_{N-2 i-2-s}(m+i) \\
& =\delta(s) \mathbf{I} \\
& \sum_{i=0}^{K-s} \mathbf{H}_{N-1-2 i}(m+i)\left[\boldsymbol{\Phi}_{N-1-2 i}^{(1)}\right]^{T}\left[\boldsymbol{\Phi}_{N-1-2 i}^{(1)}\right] \\
& \times \mathbf{H}_{N-1-2 i}(m+i) \\
& +\mathbf{H}_{N-2 i}(m+i)\left[\boldsymbol{\Phi}_{N-2 i}^{(2)}\right]^{T}\left[\boldsymbol{\Phi}_{N-2 i-s}^{(2)}\right] \mathbf{H}_{N-2 i-s}(m+i) \\
& =\delta(s) \mathbf{I}
\end{aligned}
$$

To simplify the above equations, we need to select $\left[\boldsymbol{\Phi}_{i}^{(j)}\right]_{k n}$ $(j=1,2)$, so that

$$
\left[\boldsymbol{\Phi}_{i}^{(j)}\right]^{T} \boldsymbol{\Phi}_{i+s}^{(j)}=\delta(s) \mathbf{I}
$$

It is clear that the definitions in (54)-(57) do not satisfy the condition (66). Therefore, a time-varying MDFT filter bank that has the prototype filter with limited length cannot be perfectly reconstructed. However, it is possible to get the small distortion using the window switching methods, which can be achieved through keeping the prototype window as soon as possible by switching the windows in the transient periods.

In the following we give an example to describe how to design the time-varying MDFT filter bank using window switching methods. Suppose that at time $m$ we want to switch from an MDFT filter bank with roll-off factor $r=0.5$ to another MDFT filter bank with roll-off factor $r=0.3$. The prototype windows are showed in Figures 5(c) and 5(d), respectively. We first represent the analysis matrix $\mathbf{T}_{a}(m)$ around $m$ using block diagram schematically in Figure 7.

From Figure 7, we can see that the reconstructed signal at each time point in this example is related to two analysis filter banks after this time point, which means that the filter bank change at time point $m$ has impact on the reconstructed signals at time point $m-2$ and $m-1$. Since time point $m$ the MDFT filter bank uses the new filter bank. The filter bank at time point $m$ is the bridge from the old filter bank to the new filter bank. So, we need to decide the prototype filters at $m$ in order to keep the small distortions due to the filter bank switching. According to the principle that the prototype filters in the transient period should contain the prototype filters before and after switching, we can simply keep the 
window pieces in the second part of the prototype window at time point $m$ and replace the window pieces in the first part. Using this method we get the window matrices around $m$, where the window matrix is attained through replacing the blocks in Figure 7 with the corresponding prototype windows (see Figure 8).
To show the advantage of the introduced prototype window design method in comparison with the direct switching, we define two matrices around the time index $m$ for the above example based on the $\mathbf{T}_{a}(m)$ and $\mathbf{T}_{s}(m)$ definitions in (50), (51), and the graphical representation in Figure 7 as

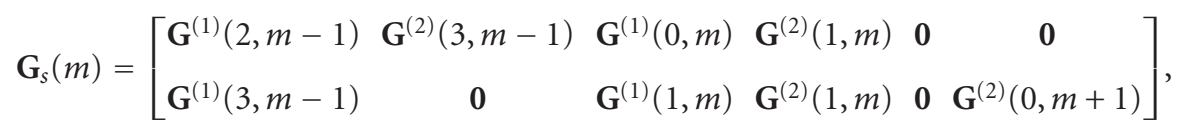

$$
\begin{aligned}
& \mathbf{P}_{s}(m) \\
& =\left[\begin{array}{ccccccccc}
\mathbf{0} & \mathbf{H}^{(1)}(0, m-1) & \mathbf{H}^{(1)}(1, m-1) & \mathbf{H}^{(1)}(2, m-1) & \mathbf{H}^{(1)}(3, m-1) & \mathbf{0} & \mathbf{0} & \mathbf{0} & \mathbf{0} \\
\mathbf{H}^{(2)}(0, m-1) & \mathbf{H}^{(2)}(1, m-1) & \mathbf{H}^{(2)}(2, m-1) & \mathbf{H}^{(2)}(3, m-1) & \mathbf{0} & \mathbf{0} & \mathbf{0} & \mathbf{0} & \mathbf{0} \\
\mathbf{0} & \mathbf{0} & \mathbf{0} & \mathbf{H}^{(1)}(0, m) & \mathbf{H}^{(1)}(1, m) & \mathbf{H}^{(1)}(2, m) & \mathbf{H}^{(1)}(3, m) & \mathbf{0} & \mathbf{0} \\
\mathbf{0} & \mathbf{0} & \mathbf{H}^{(2)}(0, m) & \mathbf{H}^{(2)}(1, m) & \mathbf{H}^{(2)}(2, m) & \mathbf{H}^{(2)}(3, m) & \mathbf{0} & \mathbf{0} & \mathbf{0} \\
\mathbf{0} & \mathbf{0} & \mathbf{0} & \mathbf{0} & \mathbf{0} & \mathbf{H}^{(1)}(0, m+1) & \mathbf{H}^{(1)}(1, m+1) & \mathbf{H}^{(1)}(2, m+1) & \mathbf{H}^{(1)}(3, m+1) \\
\mathbf{0} & \mathbf{0} & \mathbf{0} & \mathbf{0} & \mathbf{H}^{(2)}(0, m+1) & \mathbf{H}^{(2)}(1, m+1) & \mathbf{H}^{(2)}(2, m+1) & \mathbf{H}^{(2)}(3, m+1) & \mathbf{0}
\end{array}\right] .
\end{aligned}
$$

Suppose that the MDFT filter banks before and after the time point $m$ have very small alias errors which can be considered as zero. Multiplying matrices in (67) and (68), we get alias matrix

$$
\begin{aligned}
\mathbf{A}(m) & =\mathbf{G}_{s}(m) \mathbf{P}_{s}(m) \\
& =\left[\begin{array}{llllllllll}
\mathbf{0} & \mathbf{0} & \mathbf{A}_{11} & \mathbf{A}_{12} & \mathbf{A}_{13} & \mathbf{0} & \mathbf{0} & \mathbf{0} & \mathbf{0} \\
\mathbf{0} & \mathbf{0} & \mathbf{A}_{21} & \mathbf{A}_{22} & \mathbf{A}_{23} & \mathbf{0} & \mathbf{0} & \mathbf{0} & \mathbf{0}
\end{array}\right],
\end{aligned}
$$

where $\mathbf{A}_{1 i}$ and $\mathbf{A}_{2 i}(i=1,2,3)$ represent the alias errors introduced by the transition period from $m-1$ to $m$. For a perfectly reconstructed time-varying MDFT filter bank and $\mathbf{A}_{12}=\mathbf{A}_{23}=\mathbf{I}$, where $\mathbf{I}$ is the $M / 2$ unit matrix. The other alias matrices should be zero. To show that the alias errors using window switching methods are smaller than that alias errors in the direct switch, we rewrite the matrices $\mathbf{G}_{s}(m)$ and $\mathbf{P}_{s}(m)$ based on the window switching methods as

$$
\mathbf{G}_{s}^{\prime}(m)=\left[\begin{array}{lccccc}
\mathbf{G}^{(1)}(2, m-1) & \mathbf{G}^{(2)}(3, m-1) & \mathbf{G}^{(1)}(0, m-1) & \mathbf{G}^{(2)}(1, m-1) & \mathbf{0} & \mathbf{0} \\
\mathbf{G}^{(1)}(3, m-1) & \mathbf{0} & \mathbf{G}^{(1)}(1, m-1) & \mathbf{G}^{(2)}(1, m-1) & \mathbf{0} & \mathbf{G}^{(2)}(0, m-1)
\end{array}\right],
$$

$$
\begin{aligned}
& \mathbf{P}_{s}^{\prime}(m) \\
& =\left[\begin{array}{ccccccccc}
\mathbf{0} & \mathbf{H}^{(1)}(0, m-1) & \mathbf{H}^{(1)}(1, m-1) & \mathbf{H}^{(1)}(2, m-1) & \mathbf{H}^{(1)}(3, m-1) & \mathbf{0} & \mathbf{0} & \mathbf{0} & \mathbf{0} \\
\mathbf{H}^{(2)}(0, m-1) & \mathbf{H}^{(2)}(1, m-1) & \mathbf{H}^{(2)}(2, m-1) & \mathbf{H}^{(2)}(3, m-1) & \mathbf{0} & \mathbf{0} & \mathbf{0} & \mathbf{0} & \mathbf{0} \\
\mathbf{0} & \mathbf{0} & \mathbf{0} & \mathbf{H}^{(1)}(0, m-1) & \mathbf{H}^{(1)}(1, m-1) & \mathbf{H}^{(1)}(2, m) & \mathbf{H}^{(1)}(3, m-1) & \mathbf{0} & \mathbf{0} \\
\mathbf{0} & \mathbf{0} & \mathbf{H}^{(2)}(0, m-1) & \mathbf{H}^{(2)}(1, m-1) & \mathbf{H}^{(2)}(2, m-1) & \mathbf{H}^{(2)}(3, m) & \mathbf{0} & \mathbf{0} & \mathbf{0} \\
\mathbf{0} & \mathbf{0} & \mathbf{0} & \mathbf{0} & \mathbf{0} & \mathbf{H}^{(1)}(0, m+1) & \mathbf{H}^{(1)}(1, m+1) & \mathbf{H}^{(1)}(2, m+1) & \mathbf{H}^{(1)}(3, m+1) \\
\mathbf{0} & \mathbf{0} & \mathbf{0} & \mathbf{0} & \mathbf{H}^{(2)}(0, m-1) & \mathbf{H}^{(2)}(1, m+1) & \mathbf{H}^{(2)}(2, m+1) & \mathbf{H}^{(2)}(3, m+1) & \mathbf{0}
\end{array}\right]
\end{aligned}
$$


The corresponding alias error matrix reads

$$
\begin{aligned}
\mathbf{A}^{\prime}(m) & =\mathbf{G}_{s}^{\prime}(m) \mathbf{P}_{s}^{\prime}(m) \\
& =\left[\begin{array}{lllllllll}
\mathbf{0} & \mathbf{0} & \mathbf{0} & \mathbf{I} & \mathbf{A}_{13}^{\prime} & \mathbf{A}_{12}^{\prime} & \mathbf{0} & \mathbf{0} & \mathbf{0} \\
\mathbf{0} & \mathbf{0} & \mathbf{0} & \mathbf{0} & \mathbf{I} & \mathbf{A}_{23}^{\prime} & \mathbf{A}_{21}^{\prime} & \mathbf{0} & \mathbf{0}
\end{array}\right] .
\end{aligned}
$$

To measure the alias error, we define the average signal-tonoise rate (AVSNR) as

$$
\begin{aligned}
\operatorname{ASNR} & =\frac{1}{M} \sum_{i=0}^{M-1}\left\|\mathbf{p}_{i}-\mathbf{b}_{i}\right\|^{2}, \\
\mathrm{ASNR}^{\prime} & =\frac{1}{M} \sum_{i=0}^{M-1}\left\|\mathbf{p}_{i}^{\prime}-\mathbf{b}_{i}\right\|^{2},
\end{aligned}
$$

where $\mathbf{p}_{i}$ and $\mathbf{p}_{i}^{\prime}$ are the row vectors in $\mathbf{A}(m)$ and $\mathbf{A}^{\prime}(m)$, respectively. $\mathbf{b}_{i}$ is the row vector of the following matrix

$$
B=\left[\begin{array}{lllllllll}
0 & 0 & 0 & \text { I } & 0 & 0 & 0 & 0 & 0 \\
0 & 0 & 0 & 0 & I & 0 & 0 & 0 & 0
\end{array}\right] .
$$

Simulation results show that

$$
\text { ASNR }>\text { ASNR' }
$$

So, we say that the window switching design method can reduce the alias errors in the transition period than the direct switch.

The simulation is done in Matlab. The prototype filter coefficients are calculated according to (45) with roll-off factor $r=0.5$ and $r=0.3$, respectively. The filter bank coefficients are attained using (27)-(30). The MDFT filter banks before time $m$ have prototype filter with roll-off factor 0.3 . After time $m$, the prototype filter of the MDFT filter banks has the roll-off factor $r=0.5$.

Here, we have some remarks on the structure in matrices (70) and (71). The matrix $\mathbf{G}_{s}^{\prime}(m)$ is the synthesis matrix, and the matrix $\mathbf{P}_{s}^{\prime}(m)$ is the analysis matrix. In $\mathbf{G}_{s}^{\prime}(m)$ all coefficients are coming from the MDFT filter bank at time $m-1$, which means that the synthesis filter bank does not need to be redesigned during the transition period. We need just to redesign the analysis filter banks. This can reduce the design complexity. Based on the principle of window switching methods, at time $m$ the prototype window pieces in the first half part should be replaced by the prototype window in the second half part at time $m-1$. But this principle is a general principle. In the concrete application, we should make some adjustments. From Figure 3 we know that an MDFT filter bank consists of two filter banks with $M / 2$ sample delays of each other. The prototype window in the window matrix in Figure 7 has also $M / 2$ sample delays, which leads to that one piece of prototype window at time $m+1$ is involved in the filter bank operation at time $m$. So, we need to replace $\mathbf{H}^{(2)}(0, m+1)$ in (71) with $\mathbf{H}^{(2)}(0, m-$ 1) to make the matrix element $\left[\mathbf{A}^{\prime}(m)\right]_{1,4}$ in (72) equal to I. Similarly, we need to replace $\mathbf{H}^{(1)}(3, m)$ in $(71)$ with $\mathbf{H}^{(1)}(3, m-1)$ to make sure that the alias error $\left[\mathbf{A}^{\prime}(m)\right]_{0,6}=0$.

\section{Conclusion}

In this paper, we have analyzed the time-varying MDFT filter bank in detail in both frequency and time domain. The mechanism of the aliasing cancellation for the time-invariant MDFT is presented. The PR conditions for the time-varying and time-invariant MDFT filter banks are given. For the design of time-varying MDFT filter banks, we used the window switching methods. The example shows that the window switching method is simple and useful, in particular for the design of time-varying filter banks with modulation, such as time-modulated cosine filter bank and time-varying MDFT filter bank.

\section{Acknowledgment}

This work is supported by the National Natural Science Foundation of China (no. 61071195).

\section{References}

[1] N. T. Fliege, "Modified DFT polyphase SBC filter banks with almost perfect recomstruction," in Proceeding of IEEE International Conference on Acoustics, Speech and Signal Processing (ICASSP '94), April 1994.

[2] N. J. Fliege, "Closed form design of prototype filters for linear phase DFT polyphase filter banks," in Proceedings of IEEE International Symposium on Circuits and Systems, pp. 651-654, May 1994.

[3] N. J. Fliege, "Computational efficiency of modified DFT polyphase filter banks," in Proceedings of the 27th Asilomar Conference on Signals, Systems \& Computers, pp. 1296-1300, November 1993.

[4] T. Karp and N. J. Fliege, "Modified DFT filter banks with perfect reconstruction," IEEE Transactions on Circuits and Systems II, vol. 46, no. 11, pp. 1404-1414, 1999.

[5] G. Wang, "Analysis of quantization errors in subband speech coding with modified DFT filter banks," Signal Processing, vol. 86, no. 2, pp. 341-352, 2006.

[6] ISO/IEC JTCI/SC29, "Information technology-coding of moving pictures and associated audio for digital storage media at up to about 1.5 Mbit/s-IS 11172-3 (audio)," 1992.

[7] S. Shlien, "The modulated lapped transform, its time-varying forms, and its applications to audio coding standards," IEEE Transactions on Speech and Audio Processing, vol. 5, no. 4, pp. 359-366, 1997.

[8] M. G. Bellangerr and J. L. Daguet, "TDM-FDM Transmultiplexer: Digital Polyphase and FFT," IEEE Transactions on Comunnication, vol. 22, pp. 1199-1294, 1974.

[9] R. Yue, S. A. Vorobyov, and A. B. Gershman, "Adaptive OFDM techniques with one-bit-per-subcarrier channel-state feedback," IEEE Transactions on Communications, vol. 54, no. 11, pp. 1993-2003, 2006.

[10] S. Sandeep Pradhan and V. U. Reddy, "A new approach to subband adaptive filtering," IEEE Transactions on Signal Processing, vol. 47, no. 3, pp. 655-664, 1999.

[11] P. P. Vaidyanathan, "Multirate digital filters, filter banks, polyphase networks, and applications: a tutorial," Proceedings of the IEEE, vol. 78, no. 1, pp. 56-93, 1990.

[12] H. S. Malvar, Signal Processing with Lapped Transforms, Artech House, Boston, Mass, USA, 1992. 
[13] G. Wang, "Time-varying cosine-modulated filter banks," Digital Signal Processing, vol. 15, no. 3, pp. 237-254, 2005.

[14] G. Wang, "The most general time-varying filter bank and time-varying lapped transforms," IEEE Transactions on Signal Processing, vol. 54, no. 10, pp. 3775-3789, 2006.

[15] G. Wang, "Analysis of M-channel time-varying filter banks," Digital Signal Processing, vol. 18, no. 2, pp. 127-147, 2008.

[16] U. Heute, "Noise reduction," in Topics in Acoustic Echo and Noise Control, E. Haensler and G. Schmidt, Eds., chapter 9, pp. 325-384, Springer, Berlin, Germany, 2006.

[17] P. P. Vaidyanathan, Multirate Systems and Filter Banks, Signal Processing Series, Prentice-Hall, Englewood Cliffs, NJ, USA, 1993.

[18] G. Wang, "Time-varying discrete-time signal expansions as time-varying filter banks," IET Signal Processing, vol. 3, no. 5, pp. 353-367, 2009. 